\title{
Enterprise Network Packet Filtering for Mobile Cryptographic Identities
}

\author{
Janne Lindqvist and Essi Vehmersalo \\ Helsinki University of Technology
}

\author{
Jukka Manner \\ Helsinki University of Technology \\ \{janne.lindqvist,essi.vehmersalo,jukka.manner\}@tkk.fimiika@iki.fi
}

\author{
Miika Komu \\ Helsinki Insitute of Information Technology
}




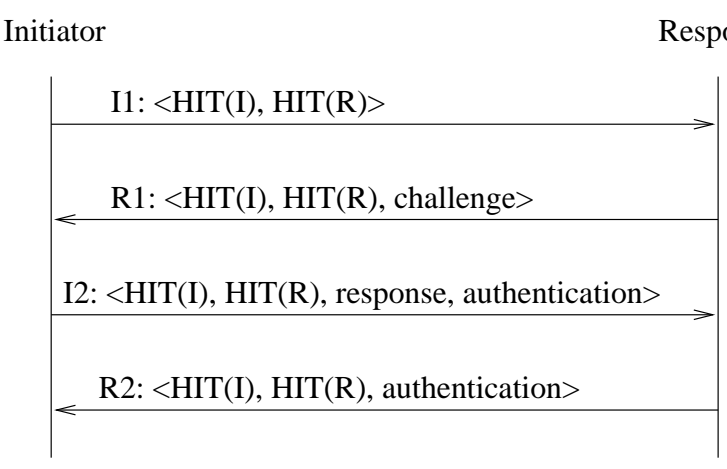

Figure 1: HIP base exchange

The firewall solution introduced in this abstract does not require Internet-wide deployment of HIP. An enterprise can deploy HIP gradually to harness the integrated security, mobility, and multihoming capabilities for employees. Services and clients that do not use HIP continue to operate with the old system.

\section{Implementation}

The firewall uses HITs as access control list identifiers, but also some other properties of network packets can be used in the firewall rules. When a connection is initiated (figure 1), the firewall verifies that the HITs of an I1 message match the access control list and it records the HITs and IP addresses of the Initiator and Responder. It is trivial for an attacker to forge these HITs, since there are no signatures to be verified at this stage. The I1 does not contain any signature, which means that the not firewall, nor the responder, can verify its authenticity. Therefore, a forged I1 can reach the responder through the firewall. However, a connection cannot be established because a verified and completed base exchange is required before data traffic is allowed into the network.

The responder sends an R1 and the firewall checks the HITs from its ACLs. This can be used to enforce access control restrictions to the Responders behind the firewall. The firewall records the HITs of the Initiator and the Responder and their IP addresses from the R1.

Upon receiving the $\mathrm{R} 1$, the Initiator solves the puzzle and sends an I 2 packet. The I 2 contains a public key and a signature calculated using the private key of the Initiator. The firewall can verify the signature either using the public key from the packet or a preconfigured public key. If the verification fails, the firewall discards the packet. Similarly, the firewall checks the response, R2, from the responder. The I2 and R2 messages contain the IPsec ESP SPI values that the firewall needs to establish state to track ESP traffic. Similarly, the firewall uses a message with the LOCATOR parameter to continue the tracking

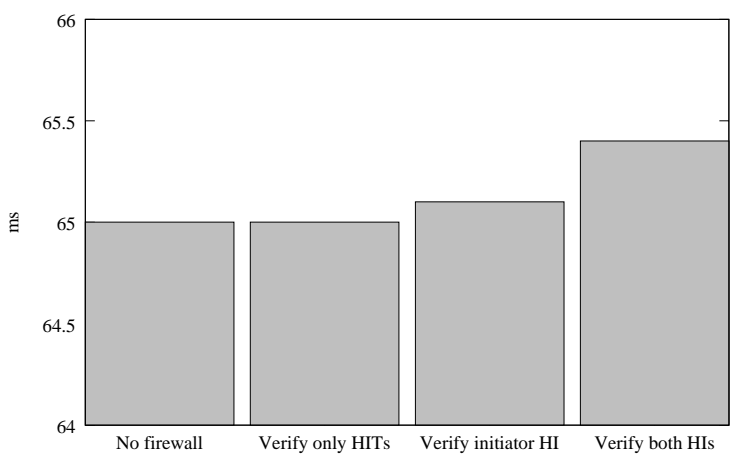

Figure 2: TCP connection establishment time

of IPsec ESP flows upon end-host IP address changing handovers. Further, the SPI state expires when there is no traffic for a certain time period. This guarantees that the state is removed when a mobile node disappears, for example, moves further away or shuts down.

We conducted a set of measurements with the firewall to get a rough idea of its performance. The evaluation environment consisted of a server and five clients. The clients were within a single network separated from the server with a combined router and firewall. All of the hosts had a single Pentium 4 processor ( $3 \mathrm{Ghz}$ ) and the IPv6 network operated at 100 Mbit speed. The Linux kernel version was 2.6.17.3. We used the 1024 bit RSA as asymmetric keys. The symmetric keys were AES (128 bits) for HIP encryption, SHA1 (160 bits) for IPsec authentication and 3DES (192 bits) for IPsec encryption.

We measured the time observed by an application to complete UNIX connect() system call, which establishes first a TCP handshake. This time was under $1 \mathrm{~ms}$ on the average without HIP. With HIP, the time was roughly 65 $\mathrm{ms}$, independently whether the HIP firewall was active or not. In addition, the use of initiator and responder signatures caused an extra delay of $1 \mathrm{~ms}$ at the maximum. The signature check was fast because the verification is quite fast in RSA. The TCP connection times are illustrated in Figure 2.

The full version of this work is available as [1].

\section{References}

[1] J. Lindqvist, E. Vehmersalo, M. Komu, and J. Manner. Enterprise Network Packet Filtering for Mobile Cryptographic Identities. Telecommunications Software and Multimedia Laboratory Research Report: TML-B8, Helsinki University of Technology, June 2007.

[2] R. Moskowitz and P. Nikander. Host identity protocol architecture. RFC 4423, IETF, May 2006. 